\title{
Domain wall generated polarity in ferroelastics: Results from resonance piezoelectric spectroscopy, piezoelectric force microscopy, and optical second harmonic generation measurements in $\mathrm{LaAlO}_{3}$ with twin and tweed microstructures
}

\author{
Hiroko Yokota $\odot,{ }^{1,2}$ C. R. S. Haines $\odot,{ }^{3, *}$ Suguru Matsumoto $\odot,{ }^{4}$ Nozomo Hasegawa $\odot,{ }^{1}$ Michael A. Carpenter, ${ }^{3}$ Yooun Heo, \\ Alexe Marin, ${ }^{5}$ E. K. H. Salje, ${ }^{3}$ and Yoshiaki Uesu $\odot^{6}$ \\ ${ }^{1}$ Department of Physics, Chiba University, 1-33 Yayoi-cho, Inage-ku, Chiba 263-8522, Japan \\ ${ }^{2}$ JST PRESTO, 4-1-8 Honcho, Kawaguchi, Saitama 332-0012, Japan \\ ${ }^{3}$ Department of Earth Sciences, University of Cambridge, Downing Street, Cambridge CB2 3EQ, United Kingdom \\ ${ }^{4}$ Department of Physics, Faculty of Science and Engineering, Chiba University, 1-33 Yayoi-cho, Inage-ku, Chiba 263-8522, Japan \\ ${ }^{5}$ Department of Physics, University of Warwick, Coventry CV4 7AL, United Kingdom \\ ${ }^{6}$ Department of Physics, Faculty of Science and Engineering, Waseda University, 3-4-1 Okubo, Shinjuku-ku, Tokyo 169-8555, Japan
}

(Received 10 April 2020; revised 21 July 2020; accepted 31 August 2020; published 29 September 2020)

\begin{abstract}
Ferroelastic $\mathrm{LaAlO}_{3}$ (space group $R \overline{3} c$ ) exists with two different microstructures: twins and tweed. Both microstructures contain electrical dipole moments. Polarity inside ferroelastic twin walls has been shown using two complementary experimental techniques and identical samples. PFM reveals a weak piezoelectric effect at the loci of the domain walls. In tweed samples, the PFM signal is finite but variable in the entire sample. PFM shows that same characteristic tweed microstructure as observed optically. The piezoelectric effect is of a similar magnitude inside twin walls and, space averaged, in the tweed microstructure (and approximately one order of magnitude smaller than in $c$-oriented $\mathrm{PbTiO}_{3}$ single crystals). Resonance piezoelectric spectroscopy proves that domain walls vibrate under the application of an external driving electric field. The resonance frequency is very close to stress induced vibrations. This is evidence for weak but finite coupling between the local dipole moments in the domain walls and the external electric field. The same coupling and the piezoelectric response are much stronger in the tweed sample. Symmetry breaking by dipolar vectors in a $\mathrm{LaAlO}_{3}$ sample with tweed has been confirmed by the observation of optical second harmonic signals. The noncentrosymmetric point group is identified as $3 m$ in agreement with earlier work on twinned $\mathrm{LaAlO}_{3}$ but in contradiction with predictions of Landau-Ginzburg theory of simple ferroelastic wall structures.
\end{abstract}

DOI: 10.1103/PhysRevB.102.104117

\section{INTRODUCTION}

High memory capacities and electrical wiring on a much finer scale than achievable with current technologies are possible when active elements in devices are not related to bulk properties but to domain walls that contain the desired functionalities [1-12]. In a first step, work was focused on exploring highly conducting domain walls as a replacement of wires in device applications. Such domain walls are designed to carry high currents and it was historically the discovery of superconducting twin walls [13] that opened a wide field of applications in "domain boundary engineering" where the domain wall is the device and not the bulk. This implied that the design of device materials depends largely on tailoring appropriate domain walls and their patterns [1,2]. Later, electric dipole moments were observed inside ferroelastic domain walls so that switchable ferroelectricity can potentially be confined to domain walls. Ferroelectric switching then does not interfere with depolarization fields and additional switching of domains in the bulk. So far, the coercive field of switching is too high for applications. The amplitude of the local dipole moment is largely sufficient

\footnotetext{
*Corresponding author: crsh2@cam.ac.uk
}

for applications $[12,14]$ although first experiments and computer simulations on $\mathrm{SrTiO}_{3}$ suggest that in this material the wall dipoles can be switched only at very low temperatures $(<40 \mathrm{~K})[15,16]$.

The length scale of an active device is restricted to the size of domain walls or to even smaller structures such as Bloch lines inside domain walls [13,14,16-20]. This approach requires-at least at the present sensitivity for the detection of ferroic functionalities - that many walls cooperate to induce a measurable macroscopic response to applied fields. The aim is, therefore, to produce high wall concentrations. The highest concentration was predicted for a tweed structure, which is a densely interwoven network of domain walls [21-26]. Tweed has another property: it will form a domain glass with a nonergodic response to external forcing. Domain glass $[27,28]$ is akin to polar nanoregions, which are known to exist in relaxor materials [29-31]. These arguments indicate that tweed is often stabilized by defects while dynamic tweed [24] exists also for very low defect concentrations [31]. It was then argued that tweed structures are polar, either via the flexoelectric effect or via bilinear coupling between the strain and local dipole moments [32-36].

In addition to $\mathrm{SrTiO}_{3}, \mathrm{BiFeO}_{3}, \mathrm{CaTiO}_{3}$, etc., with polar domain walls, $\mathrm{LaAlO}_{3}$ appears to be a promising but also 
very challenging candidate for domain boundary engineering. $\mathrm{LaAlO}_{3}$ is an improper nonpolar ferroelastic with a structural phase transition from cubic $P m \overline{3} m$ to rhombohedral $R \overline{3} c$ at 813 K [37-39]. Previous attempts to observe polar domain walls failed. The breakthrough came when Yokota et al. [40] discovered polar domain walls in $\mathrm{LaAlO}_{3}$ by optical second harmonic generation (SHG), although observations by resonant piezoelectric spectroscopy (RPS) or surface techniques like piezoelectric force microscopy (PFM) had previously failed to detect polarity.

In parallel, it was shown that highly stressed $\mathrm{LaAlO}_{3}$ crystals develop tweed microstructures and show extremely strong piezoelectricity [41]. It is the purpose of this paper to connect these two observations. By using complementary techniques, we have now confirmed polarity in twin walls and combined all observations in one conceptual framework. In this paper we present RPS and PFM results using the identical twinned sample, with no tweed, that previously showed SHG signals. Our results reveal weak piezoelectricity, which we relate to polar domain walls. We have also conducted SHG microscope observations on a tweeded $\mathrm{LaAlO}_{3}$ crystal, which was used for previous RPS experiments. For the tweeded sample an $\mathrm{SH}$ wave was generated from the bulk, in contrast with the case of twinned $\mathrm{LaAlO}_{3}$ in which $\mathrm{SH}$ activity was confined to the domain walls. These results are consistent with that fact that tweeded $\mathrm{LaAlO}_{3}$ crystals produce a larger piezoelectric signal than twinned crystals. The proposed polar point group for the piezoactive regions, $3 m$, is consistent with the SHG measurements in both tweeded and twinned $\mathrm{LaAlO}_{3}$, suggesting that the same distortion is responsible for the piezoelectricity detected in samples with both kinds of microstructure. Imaging from PFM supports the conclusion from SHG that the polarity is localized. Finally, an RPS signal demonstrates that even though the domain walls will make up a tiny fraction of the sample volume they give rise to a macroscopic piezoelectric response-in this case to vibrations of a millimeter-sized sample.

\section{EXPERIMENT}

\section{A. Sample preparation}

A commercially available $\mathrm{LaAlO}_{3}$ single crystal, hereafter the twinned $\mathrm{LaAlO}_{3}$ sample, with dimensions of $10 \times 10 \times$ $0.5 \mathrm{~mm}^{3}$ ( $\mathrm{K} \& \mathrm{R}$ Creation) was used for PFM and RPS experiments. We chose a $\left\langle\begin{array}{llll}0 & 0 & 1\end{array}\right\rangle_{\mathrm{pc}}$-oriented $\mathrm{LaAlO}_{3}$ single crystal with $\left\{\begin{array}{lll}1 & 0 & 0\end{array}\right\}_{p c}$ edges as the specimen. Here, $p c$ means the pseudocubic perovskite axis, and all crystallographic directions are referred to these axes. This sample has been characterized previously [40] and contains only twin walls (no tweed) that have been shown to be $\mathrm{SH}$ active. For the SHG measurements on tweeded $\mathrm{LaAlO}_{3}$, a 2-in. disk (MTI Corporation) of single-crystal $\mathrm{LaAlO}_{3}$ was used as the specimen. The disk was cut into a narrow strip and a mechanical stress was applied in the cutting process in the manner described in Salje et al. [41]. This produced a tweed microstructure throughout the sample. From this tweeded sample, piezoelectricity has been previously confirmed by PFM and RPS measurements.

\section{B. RPS}

In RPS experiments, mechanical resonances of a mm-sized sample are excited in a frequency range $\sim 0.1-1 \mathrm{MHz}$ by a weak AC electric field. The elastic resonances are then measured acoustically $[23,25,42]$. The technique is closely related to resonant ultrasound spectroscopy (RUS), which is generally undertaken in parallel with RPS [43-45]. The applied voltage in our experiment was $25 \mathrm{~V}$, which corresponds to an electric field across the $0.5-\mathrm{mm}$-thick sample of $50 \mathrm{kV} / \mathrm{m}$. Analysis of elastic properties was undertaken by fitting selected peaks with an asymmetric Lorentzian function to give the peak frequency $f$. The natural resonances of a mm-sized single-crystal sample involve primarily shearing motions and the variations of $f^{2}$ therefore reflect variations of different combinations of predominantly shear elastic constants. These natural resonances frequencies are determined by the geometry and the values of the single-crystal elastic constants. They are the same whether excited mechanically or electrically. The temperature dependence of these frequencies should also therefore be the same in RUS and RPS. All crystals will show resonance peaks in an RUS spectrum as long as the loss is not extremely high or the crystal cracked, but only piezoelectric crystals will show resonance peaks in an RPS spectrum.

The RPS probe was mounted in a cryostat and liquid nitrogen was used to cool the sample to $103 \mathrm{~K}$. A heater was then used to stabilize the sample at 20 set temperatures between 103 and $288 \mathrm{~K}$. The first increment was $5 \mathrm{~K}$ (so the first two points on heating were 103 and $108 \mathrm{~K}$ ). After this point the increments were $10 \mathrm{~K}$. All of these temperatures were stabilized within $1 \mathrm{~K}$ of the set point. In the data presented, the measured temperature is used rather than the set temperature and the uncertainty on these values is $0.1 \mathrm{~K}$.

\section{PFM}

PFM studies were performed using a commercial AutoProbe AP-0100 microscope from ThermoMicroscopes, working in contact mode. Piezoresponse and vertical and lateral piezoresponse force microscopy (PFM) images were obtained with an $\mathrm{AC}$ voltage of $2.0 \mathrm{~V}$ at around $312 \mathrm{kHz}$ applied to a Pt coated silicon cantilever with a spring constant of around 2 N/m (OSCM-PT-R3, Bruker). Values of a local piezoelectric coefficient have been estimated from the slope of the PFM signal versus the ac excitation signal and by comparing the slopes obtained using the same cantilever for the $\mathrm{LaAlO}_{3}$ samples and a reference $\mathrm{PbTiO}_{3}$ sample. The sample was mounted so that the scanning direction should be perpendicular to the extended twin walls. This was done by eye so a misalignment of $+/-10^{\circ}$ is possible. It has been found that scanning the tip in this way makes it more likely that the subtle features associated with a twin wall can be easily resolved.

\section{Second harmonic generation microscope}

Second harmonic generation microscope (SHGM) observations were carried out on a tweeded $\mathrm{LaAlO}_{3}$ crystal. An $\mathrm{Nd}$-doped $\mathrm{YVO}_{4}$ laser (wavelength $1064 \mathrm{~nm}$, repetition frequency $40 \mathrm{kHz}$, pulse width $10 \mathrm{~ns}$, laser energy $3.125 \mu \mathrm{J}$ ) 


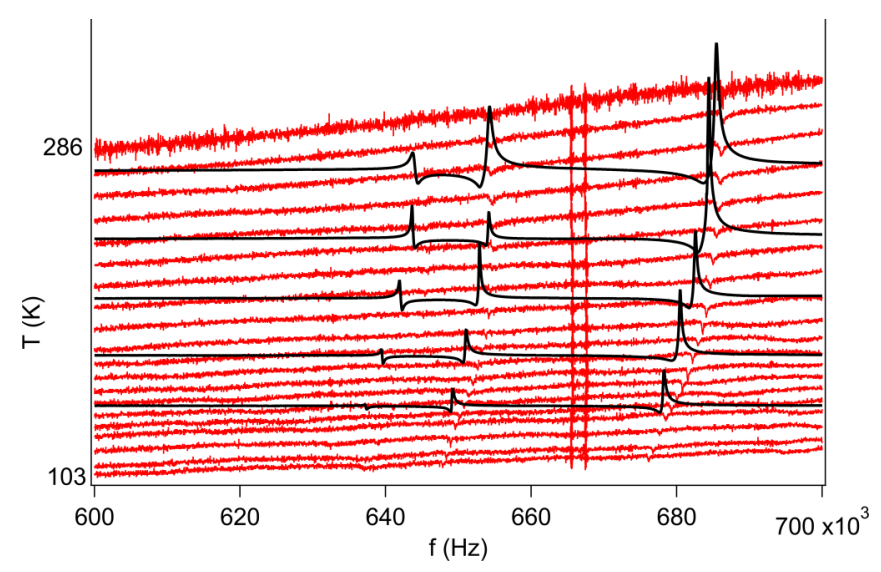

FIG. 1. Stacks of RPS (red) and RUS (black) overlain. The scaling up the $y$ axis for both is proportional to temperature. Note that there is a background on the RPS data so that the effective scaling is not quite the same for each data set, with the result that individual resonance peaks do not appear to overlap exactly.

was used as a fundamental wave. We monitored the fundamental intensity during the experiment by using a photodiode detector and this information was used to compensate the laser instability from the obtained SH intensity. Our SHG microscope was a transmission type and the confocal system had a pair of objectives with a numerical aperture (NA) of 0.7 to obtain a high spatial resolution. To construct threedimensional (3D) SH wave distribution from the specimen, a piezoactuator stage for a lateral direction ( $X Y$ plane) and a stepping-motor stage for a depth direction ( $Z$ direction) were used. Here, $X, Y$, and $Z$ directions are the laboratory axes. The SH wave was detected by a photomultiplier which was synchronized with a fundamental wave through a lock-in amplifier. It is well known that the surface produces SH waves even from centrosymmetric materials. To avoid the surface effect, the focus position was adjusted to be inside the specimen. Since the SH intensity from ferroelastic domain walls is usually extremely weak in comparison with the bulk response of normal ferroelectrics, special attention was paid to reducing the background. One of the strongest benefits of SHGM is the ability to determine the symmetry of an observed region, which is important for confirming the polar nature. We used a half wave plate to rotate the polarization direction of a fundamental wave and an analyzer to select the polarization direction of the $\mathrm{SH}$ wave.

\section{RESULTS}

\section{A. RPS}

RUS and RPS spectra of the twinned $\mathrm{LaAlO}_{3}$ are shown in Fig. 1. Strong RUS resonances in black are compared with RPS signals in red. The fact that the same resonance peaks can be seen in RPS as in RUS confirms that the crystal is piezoelectric. The temperature evolution of the resonance frequencies is shown in Fig. 2 where a close match between the RUS and RPS resonances is evident. The values of $f^{2}$, which are proportional to different combinations of the elastic constants, for the four datasets have been normalized to the value at approximately $250 \mathrm{~K}$. This is the highest temperature

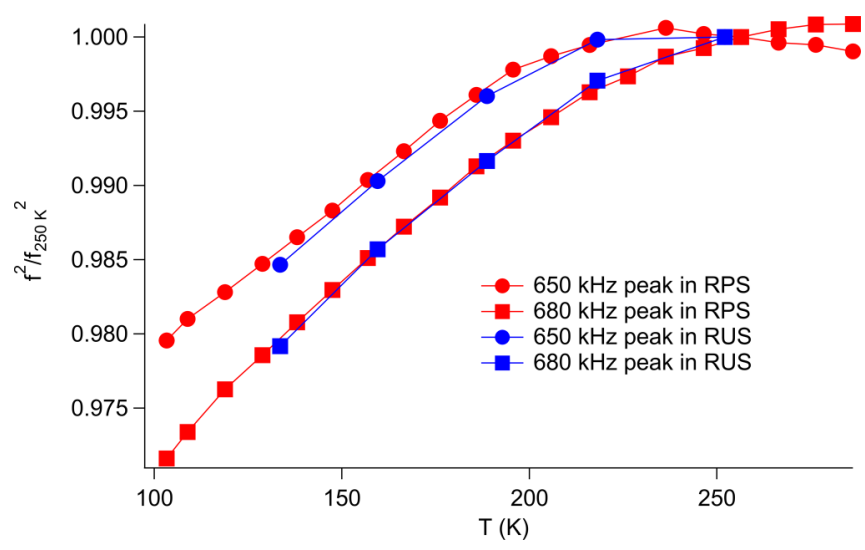

FIG. 2. $f^{2}$ normalized to the value at $250 \mathrm{~K}$ for RUS on cooling (blue) and RPS on heating (red). Closed circles are values from a peak with frequency $\sim 650 \mathrm{kHz}$ at $250 \mathrm{~K}$. Closed squares are values from a peak with frequency $\sim 680 \mathrm{kHz}$ at $250 \mathrm{~K}$.

that all four datasets extend to and allows a more obvious comparison than normalizing to an extrapolated room-temperature value.

The anomalous softening (lowering of resonance frequency) of single-crystal elastic moduli of $\mathrm{LaAlO}_{3}$ below $\sim 250 \mathrm{~K}$ was described by Carpenter et al. [46] with acoustic loss peaks which were subsequently interpreted at being due to depinning and pinning of ferroelastic domain walls [47]. The existence of the RPS signal shows that polarity exists in the twinned but nontweeded sample.

\section{B. PFM}

PFM confirmed the existence of the piezoelectric response (Fig. 3). The PFM response was not uniform but was enhanced in rather striking crossing lines of some significant width near $400 \mathrm{~nm}$ (see Fig. 3). These regions of enhanced PFM response do not correlate with the features observed in the topographic maps. We have fitted a straight line to the initial (from 0 to $2 \mathrm{~V}$ ) part of the PFM signal vs applied excitation voltage, which would be proportional to the effective piezoelectric coefficient. We then "quasicalibrated" the measurement by comparing the signals with a $c$-oriented $\mathrm{PbTiO}_{3}$ single crystal. The effective piezoelectric coefficient in $\mathrm{LaAlO}_{3}$ is about one order of magnitude lower than that of $\mathrm{PbTiO}_{3}$.

Also visible is some fine texture in the PFM signal that is not seen in the topography. This was previously reported for PFM images of polar tweed of $\mathrm{LaAlO}_{3}$. [41] This fine texture has two representative length scales: 140 and $360 \mathrm{~nm}$ (shown in Fig. 4). At present, the reason for this fine texture is unknown.

\section{SHGM}

Figure 5(a) shows the 2D image of $\mathrm{SH}$ wave distribution from the tweeded $\mathrm{LaAlO}_{3}$ sample. For comparison, an equivalent image of the twinned $\mathrm{LaAlO}_{3}$ sample is shown in Fig. 5(b). This is the same nontweeded sample used in the PFM and RPS experiments of the present study. Bright regions correspond to the areas where $\mathrm{SH}$ activity is observed. For the nontweeded $\mathrm{LaAlO}_{3}$ sample, SH-active areas 

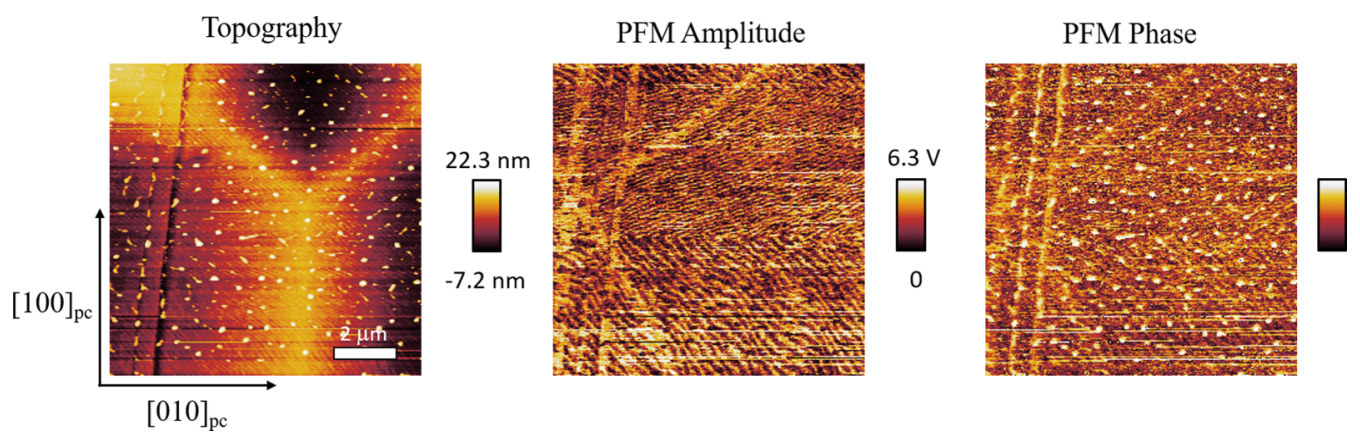

FIG. 3. AFM (left) and out-of-plane PFM amplitude (center) and phase (right) on the twinned $\mathrm{LaAlO}_{3}$ sample.

appear as straight lines under any polarization directions with a high signal-to-noise ratio (SN) of over 10:1. By comparing to polarized optical microscope images taken of the same area, we conclude that these $\mathrm{SH}$ active lines correspond to the domain walls. In contrast, the tweeded $\mathrm{LaAlO}_{3}$ sample shows $\mathrm{SH}$ activity for the whole region and relatively strong SH intensity is detected from the domain wall. This result indicates that a high density of domain walls can give rise to $\mathrm{SH}$ activity in a centrosymmetric sample. To determine the symmetry of tweeded $\mathrm{LaAlO}_{3}$, polarization dependences of the SH intensity were measured with five different configurations. First, the polarization directions of polarizer $(P)$ and analyzer $(A)$ were fixed parallel to each other and rotated together in $6^{\circ}$ steps. The $2 \mathrm{D}$ images obtained with different polarizations were then divided into several hundreds of small grids and the SH intensities summed in each of these regions. The summed-up SH intensity was plotted as a function of polarization direction across the grid to construct a polar map.
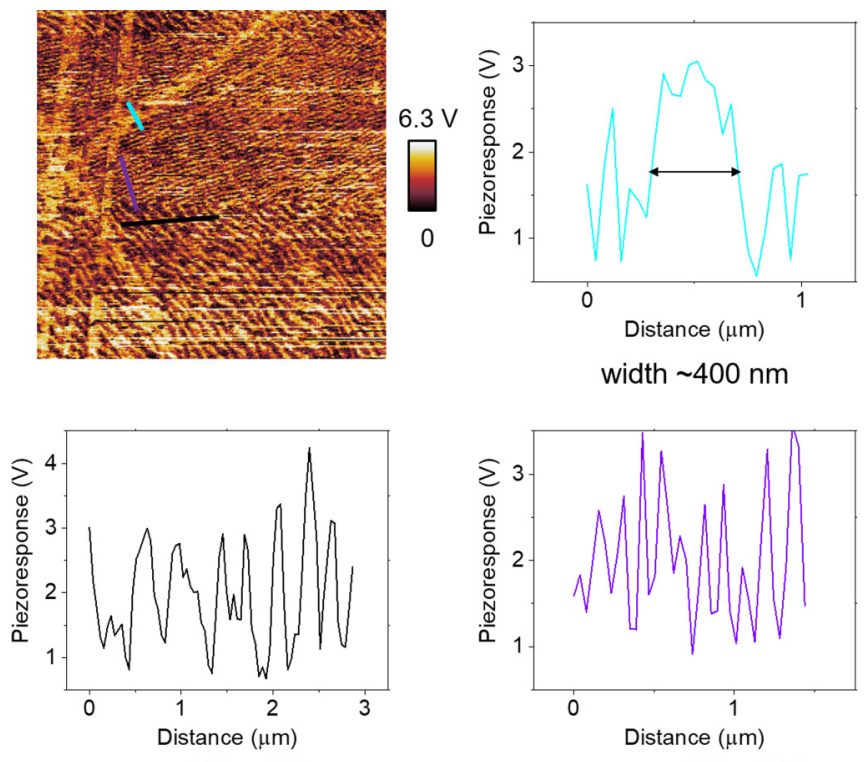

average width $\sim 360 \mathrm{~nm}$

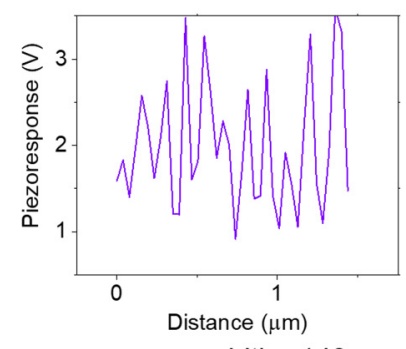

average width $\sim 140 \mathrm{~nm}$

FIG. 4. PFM response (top left) and cross sections shown in colors which match the line on the top left image to which they correspond. Top right (light blue) shows the PFM profile across an extended linelike feature of enhanced PFM response. Bottom left (black) and right (purple) show the two different length scales of the fine texture.
Figure 6(a) is the SH polar map of the same area as shown in Fig. 5(a). The SH intensity exhibits strong anisotropy with almost the same pattern across the whole region. The form of the anisotropy is given in Fig. 6(b) which displays four local maxima $90^{\circ}$ apart. The directions of the local maxima do not coincide with that of the domain wall and are inclined by around $42^{\circ}$ from the direction of the domain wall. These directions are almost parallel to $X$ and $Y$. The $Y$ direction is the direction of the trigonal axis, the $X$ direction is in the pseudohexagonal basal plane. Measuring the SH intensity under the condition of $P$ and $A$ parallel to each other provides direct information about symmetry that exists along the propagation direction. Although it is a powerful technique for understanding the symmetry of a material, it is not sufficient to determine the crystallographic point group, especially when the orientation of the crystallographic axes is unknown.

For further understanding, the polarization dependencies of SH intensity were collected under four more conditions: (i) $P$ fixed along the $X$ direction, (ii) $P$ fixed along the $Y$ direction, (iii) $A$ fixed along the $X$ direction, and (iv) $A$ fixed along the $Y$ direction. Either $P$ or $A$ was rotated in $6^{\circ}$ steps and $2 \mathrm{D}$ images of SH wave distributions were measured to construct the polar map. Figures 7(a)-7(d) show the expanded plots of polarization dependencies for each configuration. They exhibit anisotropy with different shapes.

\section{SHG anisotropy}

For the nontweeded $\mathrm{LaAlO}_{3}$ sample, we observed SH activity from domain walls and determined the point group of the walls to being trigonal $3 m$. We therefore assumed that the point group of tweeded $\mathrm{LaAlO}_{3}$ is trigonal $3 m$ and fitted the experimental data. In point group $3 m$ there are eight nonzero nonlinear $d$ tensor components, some of which are related through symmetry. After taking into account the symmetry, the number of independent $d$ tensor component becomes 4 and the $d$ tensor matrix can be expressed in Voigt notation as

$$
\left(\begin{array}{cccccc}
\dot{d_{22}} & \dot{d_{22}} & \dot{\cdot} & \dot{d_{15}} & d_{15} & -2 d_{22} \\
d_{31} & \dot{d}_{31} & \dot{d}_{33} & \cdot & \cdot & \cdot
\end{array}\right) .
$$

In the case of a transparent crystal, Kleinman's law commonly applies and $d_{15}$ becomes equal to $d_{31}$ for $3 \mathrm{~m}$. Thus, only three $d$ tensor components remain as independent. The ratio of these three components should be the same thorough all configurations, so we fixed their values and fit the data under the constrained symmetry condition. The fitting results are 

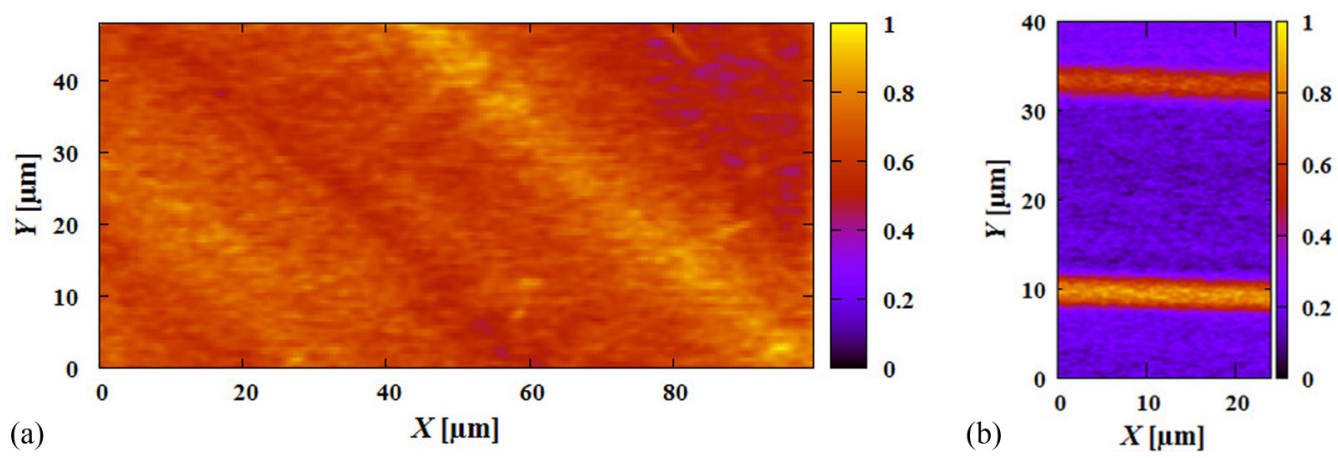

FIG. 5. 2D images of SH wave distribution under the polarization directions of polarizer and analyzer being parallel each other. Panel (a) is for the tweeded $\mathrm{LaAlO}_{3}$ sample and (b) is for the twinned $\mathrm{LaAlO}_{3}$ sample. In the case of the tweeded sample, the whole region is SH active. On the other hand, only domain walls are $\mathrm{SH}$ active in the twinned sample.

shown as red lines in Fig. 7. All five anisotropies can be well explained by point group $3 m$. For comparison, point group 32 was also examined but did not reproduce the experimental results. Since point group $3 m$ belongs to the polar class, we conclude that tweeded $\mathrm{LaAlO}_{3}$ has a polar nature and that applying a stress can produce a high-density polar texture in a nonpolar material. Note that ferroelastic walls $w$ and $w^{\prime}$ show internal symmetries according to the point groups $m$ and 2 and not the higher symmetry $3 m$ [48]. The observation of the

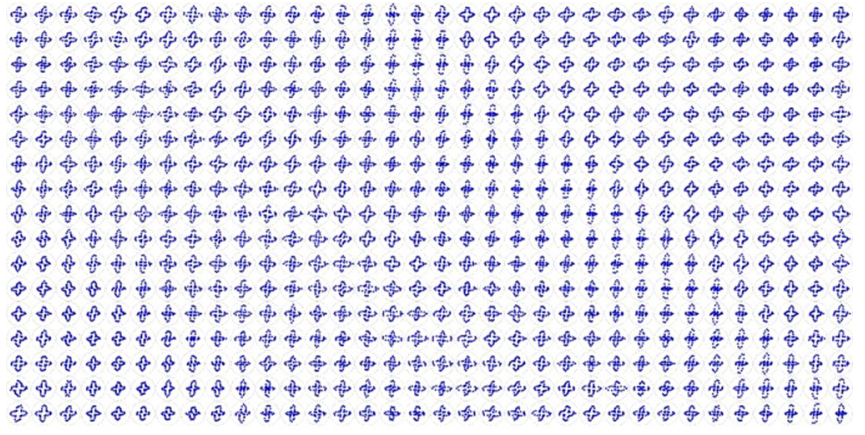

(a)

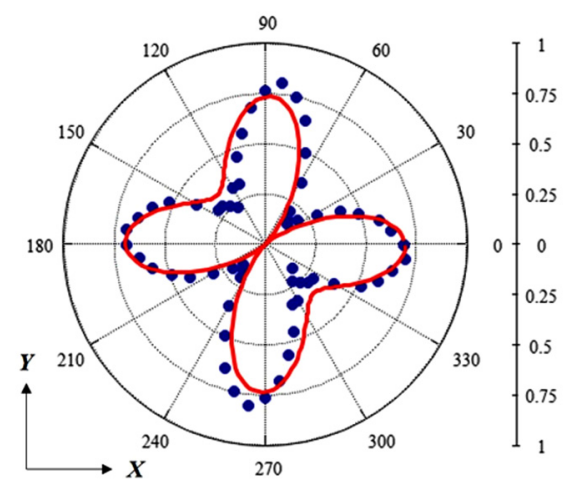

(b)

FIG. 6. (a) Polar diagram map for the tweeded $\mathrm{LaAlO}_{3}$ sample. The polarization directions of a fundamental and $\mathrm{SH}$ waves are parallel to each other and were rotated in steps of $6^{\circ}$. The sample exhibits SH anisotropy with the same form across the whole area. (b) is the expanded polarization dependence of SH intensity. Blue dots correspond to the experimental data and the red line corresponds to the result from a fit using point group $3 m$. higher symmetry in $\mathrm{LaAlO}_{3}$ together with the orientation of the domain walls according to the direction of the trigonal axis and the strong anomalous softening of the RPS signal at low temperatures indicates that the domain walls show a much more complex behavior than expected in ferroelastics.

\section{CONCLUSIONS}

We have presented results obtained from measurements of separate single-crystal samples of $\mathrm{LaAlO}_{3}$ in which the microstructure was either dominated by twin walls or by stress induced tweed. These measurements, when combined with those known from the literature [40,41], prove that the twin walls are indeed polar. Our new SHG measurements demonstrate that a piezoelectric response in tweeded $\mathrm{LaAlO}_{3}$ is present throughout the crystal. This is in agreement with previous PFM data showing a significant PFM response across the surface and from RPS [41] in which a strong resonant response was observed. Likewise, the existing SHG measurements on twinned $\mathrm{LaAlO}_{3}$ without tweed show highly localized regions of polarization confined to the twin walls [40]. This is in good agreement with the weak resonance observed in the bulk RPS data. PFM in the same sample also shows a localized response. The polar nature of $\mathrm{LaAlO}_{3}$ is due to the domain walls. In twinned $\mathrm{LaAlO}_{3}$ this means that it is highly localized to the twin walls. In tweeded $\mathrm{LaAlO}_{3}$ the microstructure consists of many widespread interfaces and the polar nature is widespread throughout the sample. The much greater volume fraction of interfaces is the principal reason that a tweeded crystal will show a much greater degree of bulk piezoelectric response than will a sample containing only simple twinning.

The comparison between twin and tweed samples shows that all polarity in twinned samples is confined to the twin walls. In tweed samples, the polarity is much more uniform and not confined to nanosized patches in the sample. A rough estimate for the piezoeffect can be based on the assumption that only the rim between tweed regions with diameters of some micrometers is piezoelectric and that the macroscopic piezoeffect is, roughly, the superposition of local piezoeffects. An open question remains that the local twin wall symmetry in ferroelastics is predicted to be either 2 or $m$. Here we confirmed the previous symmetry assessment of twin walls in $\mathrm{LaAlO}_{3}$, which is identical in tweed samples, to be $3 m$. This 


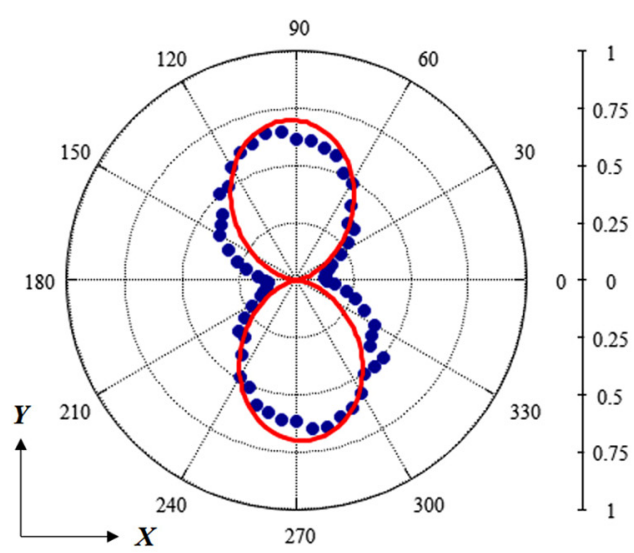

(a)

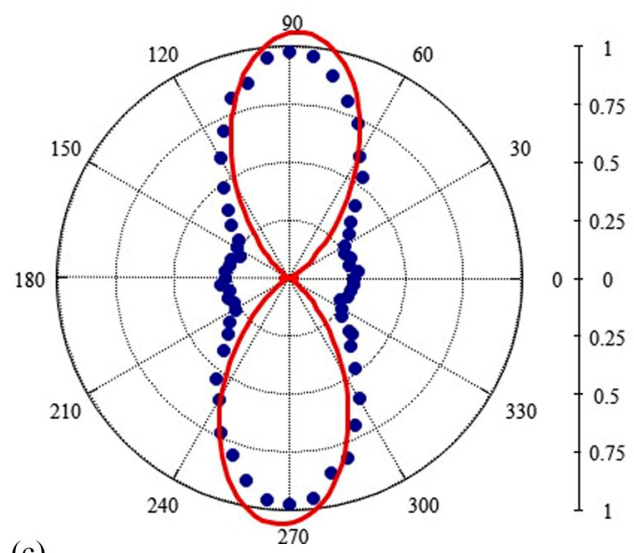

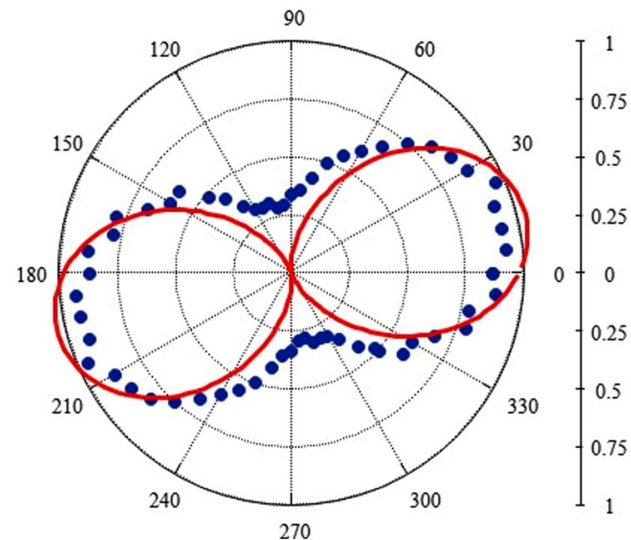

(b)

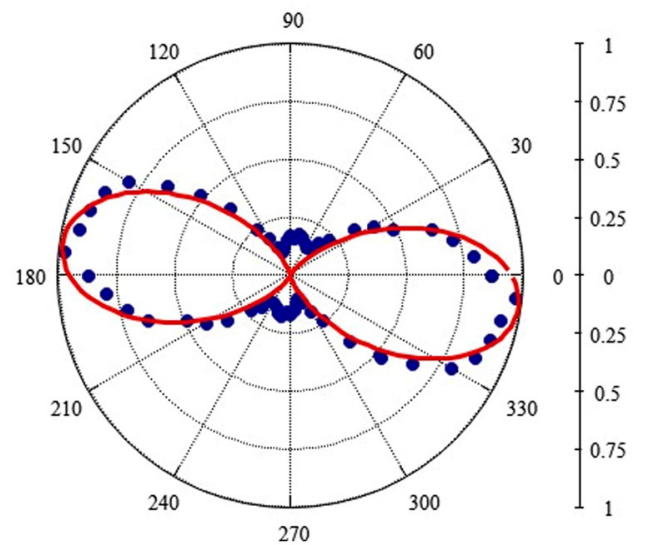

(d)

FIG. 7. Polarization dependences of SH intensity under different configurations for the tweeded sample: (a) the polarization direction of a fundamental wave fixed along the $X$ direction, (b) the polarization direction of a fundamental wave fixed along the $Y$ direction, (c) the polarization direction of an SH wave fixed along the $X$ direction, and (d) the polarization direction of an SH wave fixed along the $Y$ direction. Blue points are experimental data and red lines correspond to the fitting results, assuming point group $3 \mathrm{~m}$.

high symmetry suggests some specific atomic twin structure that remains so far enigmatic. Its solution is encouraged for future research using, e.g., high-resolution transmission electron microscopy.

\section{ACKNOWLEDGMENTS}

H.Y. gratefully acknowledges the JPSJ KAKENHI in Japan for financial support (Grant No. 17K05489), JST PRESTO Grant No. JPMJPR19LA, Japan and the Iketani Science and
Technology Foundation (Grant No. 0311045-A). This work was funded by EPSRC Grant No. EP/P024904/1, which is gratefully acknowledged. RUS facilities in Cambridge were established through grants from the Natural Environment Research Council (Grants No. NE/B505738/1 and No. NE/F017081/1) and the Engineering and Physical Sciences Research Council (Grant No. EP/I036079/1) to M.A.C. M. A. is grateful to the EPSRC for support (Grant No. EP/P031544/1).
[1] E. K. H. Salje, Multiferroic domain boundaries as active memory devices: trajectories towards domain boundary engineering, Chem. Phys. Chem. 11, 940 (2010).

[2] E. Salje and H. Zhang, Domain boundary engineering, Phase Transitions 82, 452 (2009).

[3] E. K. H. Salje, D. J. Safarik, K. A. Modic, J. E. Gubernatis, J. C. Cooley, R. D. Taylor, B. Mihaila, A. Saxena, T. Lookman, J. L. Smith, R. A. Fisher, M. Pasternak, C. P. Opeil, T. Siegrist, P. B. Littlewood, and J. C. Lashley, Tin telluride: a weakly co-elastic metal, Phys. Rev. B 82, 184112 (2010).
[4] J. Seidel, P. Maksymovych, Y. Batra, A. Katan, S.-Y. Yang, Q. He, A. P. Baddorf, S. V Kalinin, C.-H. Yang, J.-C. Yang, Y.-H. Chu, E. K. H. Salje, H. Wormeester, M. Salmeron, and R. Ramesh, Domain Wall Conductivity in La-Doped $\mathrm{BiFeO}_{3}$, Phys. Rev. Lett. 105, 197603 (2010).

[5] J. Seidel, L. W. Martin, Q. He, Q. Zhan, Y. H. Chu, A. Rother, M. E. Hawkridge, P. Maksymovych, P. Yu, M. Gajek, N. Balke, S. V. Kalinin, S. Gemming, F. Wang, G. Catalan, J. F. Scott, N. A. Spaldin, J. Orenstein, and R. Ramesh, Conduction at domain walls in oxide multiferroics, Nat. Mater. 8, 229 (2009). 
[6] G. Catalan, J. Seidel, R. Ramesh, and J. F. Scott, Domain wall nanoelectronics, Rev. Mod. Phys. 84, 119 (2012).

[7] A. Schilling, D. Byrne, G. Catalan, K. G. Webber, Y. A. Genenko, G. S. Wu, J. F. Scott, and J. M. Gregg, Domains in ferroelectric nanodots, Nano Lett. 9, 3359 (2009).

[8] S. Farokhipoor and B. Noheda, Conduction through $71^{\circ}$ domain Walls in $\mathrm{BiFeO}_{3}$ Thin Films, Phys. Rev. Lett. 107, 127601 (2011).

[9] G. Catalan, A. Janssens, G. Rispens, S. Csiszar, O. Seeck, G. Rijnders, D. H. A. Blank, and B. Noheda, Polar Domains in Lead Titanate Films under Tensile Strain, Phys. Rev. Lett. 96, 127602 (2006).

[10] D. D. Viehland and E. K. H. Salje, Domain boundarydominated systems: adaptive structures and functional twin boundaries, Adv. Phys. 63, 267 (2014).

[11] D. Wang, E. K. H. Salje, S. B. Mi, C. L. Jia, and L. Bellaiche, Multidomains made of different structural phases in multiferroic $\mathrm{BiFeO}_{3}$ : a first-principles-based study, Phys. Rev. B 88, 134107 (2013).

[12] J. F. Scott, E. K. H. Salje, and M. A. Carpenter, Domain Wall Damping and Elastic Softening in $\mathrm{SrTiO}_{3}$ : Evidence for Polar Twin Walls, Phys. Rev. Lett. 109, 187601 (2012).

[13] A. Aird and E. K. H. Salje, Sheet superconductivity in twin walls: experimental evidence of $\mathrm{WO}_{3-X}$, J. Phys.: Condens. Matter 10, L377 (1998).

[14] S. Van Aert, S. Turner, R. Delville, D. Schryvers, G. Van Tendeloo, and E. K. H. Salje, Direct observation of ferrielectricity at ferroelastic domain boundaries in $\mathrm{CaTiO}_{3}$ by electron microscopy, Adv. Mater. 24, 523 (2012).

[15] Y. Frenkel, N. Haham, Y. Shperber, C. Bell, Y. Xie, Z. Chen, Y. Hikita, H. Y. Hwang, E. K. H. Salje, and B. Kalisky, Imaging and tuning polarity at $\mathrm{SrTiO}_{3}$ domain walls, Nat. Mater. 16, 1203 (2017).

[16] T. Zykova-Timan and E. K. H. Salje, Highly mobile vortex structures inside polar twin boundaries in $\mathrm{SrTiO}_{3}$, Appl. Phys. Lett. 104, 082907 (2014).

[17] H. Yokota, H. Usami, R. Haumont, P. Hicher, J. Kaneshiro, E. K. H. Salje, and Y. Uesu, Direct evidence of polar nature of ferroelastic twin boundaries in $\mathrm{CaTiO}_{3}$ obtained by second harmonic generation microscope, Phys. Rev. B 89, 144109 (2014).

[18] L. Goncalves-Ferreira, S. A. T. Redfern, E. Artacho, and E. K. H. Salje, Ferrielectric Twin Walls in $\mathrm{CaTiO}_{3}$, Phys. Rev. Lett. 101, 097602 (2008).

[19] J. F. Scott, E. K. H. H. Salje, M. A. Carpenter, O. Aktas, M. A. Carpenter, V. V. Laguta, and J. F. Scott, Domains within Domains and Walls within Walls: Evidence for Polar Domains in Cryogenic $\mathrm{SrTiO}_{3}$, Phys. Rev. Lett. 111, 247603 (2013).

[20] E. K. H. Salje and J. F. Scott, Ferroelectric bloch-line switching: a paradigm for memory devices?, Appl. Phys. Lett. 105, 252904 (2014).

[21] A. M. Bratkovsky, S. C. Marais, V. Heine, and E. K. H. Salje, The theory of fluctuations and texture embryos in structural phase transitions mediated by strain, J. Phys.: Condens. Matter 6, 3679 (1994).

[22] K. Parlinski, V. Heine, and E. K. H. Salje, Origin of tweed texture in the simulation of a cuprate superconductor, J. Phys.: Condens. Matter 5, 497 (1993).

[23] O. Aktas, E. K. H. Salje, S. Crossley, G. I. Lampronti, R. W. Whatmore, N. D. Mathur, and M. A. Carpenter, Ferroelectric precursor behavior in $\mathrm{PbSc}_{0.5} \mathrm{Ta}_{0.5} \mathrm{O}_{3}$ detected by field-induced resonant piezoelectric spectroscopy, Phys. Rev. B 88, 174112 (2013).

[24] E. K. H. Salje, Tweed, twins, and holes, Am. Mineral. 100, 343 (2015).

[25] O. Aktas, M. A. Carpenter, and E. K. H. Salje, Polar precursor ordering in $\mathrm{BaTiO}_{3}$ detected by resonant piezoelectric spectroscopy, Appl. Phys. Lett. 103, 142902 (2013).

[26] S. Kartha, J. A. Krumhansl, J. P. Sethna, and L. K. Wickham, Disorder-driven pretransitional tweed pattern in martensitic transformations, Phys. Rev. B 52, 803 (1995).

[27] E. K. H. Salje, X. Ding, and O. Aktas, Domain glass, Phys. Status Solidi Basic Res. 251, 2061 (2014).

[28] E. K. H. Salje and M. A. Carpenter, Domain glasses: twin planes, bloch lines, and bloch points, Phys. Status Solidi Basic Res. 252, 2639 (2015).

[29] D. Viehland, M. Wuttig, and L. E. Cross, The glassy behavior of relaxor ferroelectrics, Ferroelectrics 120, 71 (1991).

[30] W. Kleemann, The relaxor enigma - charge disorder and random fields in ferroelectrics, J. Mater. Sci. 41, 129 (2006).

[31] P. Lloveras, T. Castán, A. Planes, and A. Saxena, Precursor nanoscale textures in ferroelastic martensites, Springer Ser. Mater. Sci. 148, 227 (2011).

[32] G. Catalan, A. Lubk, A. H. G. Vlooswijk, E. Snoeck, C. Magen, A. Janssens, G. Rispens, G. Rijnders, D. H. A. Blank, and B. Noheda, Flexoelectric rotation of polarization in ferroelectric thin films, Nat. Mater. 10, 963 (2011).

[33] S. Conti, S. Müller, A. Poliakovsky, and E. K. H. Salje, Coupling of order parameters, chirality, and interfacial structures in multiferroic materials, J. Phys.: Condens. Matter 23, 142203 (2011).

[34] B. Houchmandzadeh, J. Lajzerowicz, and E. Salje, Order parameter coupling and chirality of domain walls, J. Phys.: Condens. Matter 3, 5163 (1991).

[35] M. Stengel, Flexoelectricity from density-functional perturbation theory, Phys. Rev. B 88, 174106 (2013).

[36] D. Pesquera, M. A. Carpenter, and E. K. H. Salje, Glasslike Dynamics of Polar Domain Walls in Cryogenic $\mathrm{SrTiO}_{3}$, Phys. Rev. Lett. 121, 235701 (2018).

[37] C. J. Howard, B. J. Kennedy, and B. C. Chakoumakos, Neutron powder diffraction study of rhombohedral rare-earth aluminates and the rhombohedral to cubic phase transition, J. Phys.: Condens. Matter 12, 349 (2000).

[38] H. Lehnert, H. Boysen, J. Schneider, F. Frey, D. Hohlwein, P. Radaelli, and H. Ehrenberg, A powder diffraction study of the phase transition in $\mathrm{LaAlO}_{3}, \mathrm{Z}$. Kristallogr. 215, 536 (2000).

[39] S. A. Hayward, F. D. Morrison, S. A. T. Redfern, E. K. H. Salje, J. F. Scott, K. S. Knight, S. Tarantino, A. M. Glazer, V. Shuvaeva, P. Daniel, M. Zhang, and M. A. Carpenter, Transformation processes in $\mathrm{LaAlO}_{3}$ : neutron diffraction, dielectric, thermal, optical, and raman studies, Phys. Rev. B 72, 054110 (2005).

[40] H. Yokota, S. Matsumoto, E. K. H. Salje, and Y. Uesu, Symmetry and three-dimensional anisotropy of polar domain boundaries observed in ferroelastic $\mathrm{LaAlO}_{3}$ in the complete absence of ferroelectric instability, Phys. Rev. B 98, 104105 (2018).

[41] E. K. H. Salje, M. Alexe, S. Kustov, M. C. Weber, J. Schiemer, G. F. Nataf, and J. Kreisel, Direct observation of polar tweed in $\mathrm{LaAlO}_{3}$, Sci. Rep. 6, 27193 (2016). 
[42] O. Aktas and E. K. H. Salje, Macroscopic symmetry breaking and piezoelectricity in relaxor ferroelectric lead magnesium niobate, Appl. Phys. Lett. 113, 202901 (2018).

[43] E. K. H. Salje, M. A. Carpenter, G. F. Nataf, G. Picht, K. Webber, J. Weerasinghe, S. Lisenkov, and L. Bellaiche, Elastic excitations in $\mathrm{BaTiO}_{3}$ single crystals and ceramics: mobile domain boundaries and polar nanoregions observed by resonant ultrasonic spectroscopy, Phys. Rev. B 87, 014106 (2013).

[44] M. A. Carpenter, J. F. J. Bryson, G. Catalan, S. J. Zhang, and N. J. Donnelly, Elastic and anelastic relaxations in the relaxor ferroelectric $\mathrm{Pb}\left(\mathrm{Mg}_{1 / 3} \mathrm{Nb}_{2 / 3}\right) \mathrm{O}_{3}$ : II. strainorder parameter coupling and dynamic softening mechanisms, J. Phys.: Condens. Matter 24, 045902 (2012).
[45] M. A. Carpenter, Static and dynamic strain coupling behaviour of ferroic and multiferroic perovskites from resonant ultrasound spectroscopy, J. Phys.: Condens. Matter 27, 263201 (2015).

[46] M. A. Carpenter, A. Buckley, P. A. Taylor, R. E. A. McKnight, and T. W. Darling, Elastic relaxations associated with the transition in $\mathrm{LaAlO}_{3}$ : IV. an incipient instability below room temperature, J. Phys.: Condens. Matter 22, 035406 (2010).

[47] S. Kustov, I. Liubimova, and E. K. H. Salje, LaAlO: a substrate material with unusual ferroelastic properties, Appl. Phys. Lett. 112, 042902 (2018).

[48] E. K. H. Salje, Ferroelastic materials, Annu. Rev. Mater. Res. 42, 265 (2012). 